\title{
A Three-Dimensional Simulation of Particle Distribution in a Separator and Structure Optimization with the Statistical Approach of Taguchi Method
}

\author{
Jinjin Liu $\mathbb{D}^{1}{ }^{1}$ Kai Liu, ${ }^{1}$ Tong Zhao $\mathbb{D}^{1},{ }^{1}$ and Zhuofei Xu $\mathbb{D}^{2}$ \\ ${ }^{1}$ School of Mechanical and Precision Instrument Engineering, Xian University of Technology, Xian 710048, China \\ ${ }^{2}$ Faculty of Printing Packaging Engineering and Digital Media Technology, Xian University of Technology, Xi'an 710048, China \\ Correspondence should be addressed to Tong Zhao; tongzhao@xaut.edu.cn
}

Received 4 June 2018; Revised 16 October 2018; Accepted 24 October 2018; Published 27 November 2018

Guest Editor: Arshad Afzal

Copyright (C) 2018 Jinjin Liu et al. This is an open access article distributed under the Creative Commons Attribution License, which permits unrestricted use, distribution, and reproduction in any medium, provided the original work is properly cited.

\begin{abstract}
A three-dimensional numerical simulation combining discrete phase method (DPM) and porous media based on the theory of Euler-Lagrange has been employed to investigate particles distribution in a separator. The DPM model is applied to monitor the movement of individual particles and calculate the contact force between them in the separator. The simulation results display the migration feature of dust particles over time and the distribution of particles on the surface element in porous region and reveal that the flow field influences the distribution uniformity of the particles in porous area directly. Based on the analysis, the structure of separator is optimized by the Taguchi method. An orthogonal relation motion has been established. The optimal solution is achieved by the calculation of the weight relationship. The calculated optimal structure is evaluated by the signal to noise (SNR). The result reveals that the values of SNR in case are eligible. As a result, the research of the separator points out a useful and improvable method for the parameter optimization of structure design.
\end{abstract}

\section{Introduction}

Coal, as the primal fossil fuel, has played an important role in national economy, such as the areas of electricity, heating, and transportation. Coal resource can be divided into high rank coal and low rank coal. Among them, low rank coal is not only characterized by the high moisture content but also the high volatility which can cause an explosion easily [1]. Meanwhile, high temperature gas containing dust is generated during burning, which causes serious pollution. Therefore, particles elimination is necessary before blowdown. Separator is important equipment in the filtration and removal process of low rank coal. Its core component is the filter core, which has the characteristics of a high temperature resistance and pressure resistance, strong corrosion resistance, and high filtration efficiency.

In this paper, multiphase flow model is chosen for simulation analysis of the model combined with computational fluid dynamics (CFD). The commonly used mathematical models that have been raised so far can be grouped into two categories: one is the continuum-continuum approach, as represented by Euler-Euler model [2,3], and the other is the continuum discrete approach [4] represented by the EulerLagrange model. The flow field of gas is treated as a continuum phase by Navies-Stokes equations in computational cell scale while the flow field of particles is treated as a discrete phase based on DPM. The physical status of individual particles, such as the trajectory in space, is obtained by the calculation of continuum phase of the air. A two-phase model of gas-solid flow is used to simulate the coupling of porous media module.

Many scholars have studied the porous media model in the different structure. Wu [5] and Boysen [6] simulated the process of adsorption in porous. Yu Liming et al. [7] used CFD-DEM to simulate the process of pressure variation during filtration process. Wang et al. [8] researched the separation efficiency of particles with different sizes in the gas-solid flow. Zhao Tong et al. [9] researched the gas-solid flow whose particle movement and distribution are in a down-flow fluidized bed with CFD-DEM. M. Haim et al. 
[10] researched the particle-gas flows whose effect of the inlet conditions is on the numerical solutions. Xi Gao et al. [11] made a research on the evaluation of an enhanced filtered drag model compared with other eight different drag models in the three-dimensional simulations of gas-solid fluidized beds.

However, minimum studies considered the more uniformity of particles in porous media by optimizing the structural parameters. Optimization design methods have used various metaheuristics such as nonlinear search optimization [12], the Taguchi method.

The Taguchi method has been proposed by Genichi Taguchi $[13,14]$ and widely used in many fields. Rout [15] applied the Taguchi method to find the optimal setting of parameters in a manipulator and increase the positional accuracy. T. Mothilal et al. [16] applied the Taguchi method to optimize the operational parameters including the solid particle diameter, inlet air velocity, and inlet air temperature in a cyclone separator.

In summary, the paper is organized as follows: Section 2 introduces the basic theory of CFD, Euler-Lagrange Model, and the porous medium model as well; then the result of numerical simulation is discussed in a three-dimensional model of separator such as the migration distribution diagram of particles over time and the velocity distribution in filter area. Section 3 evaluates the model by using the statistics method of Taguchi to determine the weight of different structure parameters and get an effective improved plan.

\section{The Approach of Modeling}

2.1. Computational Fluid Theory. Computational fluid dynamics method is used in this study. Its basic approach is dividing the continuous physics region into discrete. Based on the Navier-Stokes equations, the fluid domain is calculated to obtain the key physical parameters of heated air such as speed, temperature, pressure, and flow positions.

The flow condition can be judged from the Reynolds number. The turbulence model is selected which includes the conversation laws of mass, momentum, and energy. The turbulence equation should be considered. Thus $\mathrm{k}-\varepsilon$ model is taken as follows:

$$
\begin{aligned}
\frac{\partial(\rho k)}{\partial t}+\frac{\partial\left(\rho k u_{i}\right)}{\partial x_{i}}= & \frac{\partial}{\partial x_{j}}\left[\left(\mu+\frac{\mu}{\sigma_{k}}\right) \frac{\partial k}{\partial x_{j}}\right]+G_{k}+G_{b} \\
& -\rho \varepsilon-Y_{M}+S_{k} \\
\frac{\partial(\rho \varepsilon)}{\partial t}+\frac{\partial\left(\rho \varepsilon u_{i}\right)}{\partial x_{i}}= & \frac{\partial}{\partial x_{j}}\left[\left(\mu+\frac{\mu}{\sigma_{k}}\right) \frac{\partial k}{\partial x_{j}}\right]+G_{1 \varepsilon} \frac{\varepsilon}{k} \\
& \cdot\left(G_{k}+C_{3 \varepsilon} G_{b}\right)-G_{2 \varepsilon} \rho \frac{\varepsilon^{2}}{k}+S_{\varepsilon}
\end{aligned}
$$

Here $\mathrm{k}$ is turbulent kinetic energy in (1) and (2). $\varepsilon$ means the dissipation rating. And we can know that Gk and Gb are the turbulent kinetic energy generation introduced by mean velocity gradient and buoyancy. $Y_{M}$ is the contribution in the turbulent pulsating pressure expansion. $C_{1 \varepsilon}, C_{2 \varepsilon}$, and $C_{3 \varepsilon}$ are empirical constants. $\sigma_{k}$ and $\sigma_{\varepsilon}$ are Prandtl numbers to $\mathrm{k}$ and $\varepsilon$ correspondingly. $S_{k}$ and $S_{\varepsilon}$ are user defined options.

2.2. The Porous Medium Model. The filter area in separator needs to be calculated with model of porous media which is defined as laminar. A flow resistance value is introduced. The porous medium model based on superficial velocity can simulate pressure loss. The flow resistance of porous material in the fluid domain is calculated by adding a feature in momentum equation. The feature is composed of two parts, Darcy viscous resistance terms and inertial loss terms, which is expressed as

$$
S_{j}=-\left(\sum_{j=1}^{3} D_{i j} \mu v_{j}+\sum_{j=1}^{3} C_{i j} \frac{1}{2} \rho|v| v_{j}\right)
$$

Here $D_{i j}$ and $C_{i j}$ is the viscous resistance and inertia loss coefficient matrix, respectively. This negative result leads to a pressure drop in the unit of porous medium. $\mu$ is the fluid viscosity coefficient. The viscous resistance and inertial resistance is calculated by the following formula (4) and (5):

$$
\begin{aligned}
\frac{1}{\alpha} & =\frac{150}{D_{p}^{2}} \frac{(1-\varepsilon)^{2}}{\varepsilon^{3}} \\
C_{2} & =\frac{3.5}{D_{p}} \frac{(1-\varepsilon)^{2}}{\varepsilon^{3}}
\end{aligned}
$$

where $D_{p}$ is the particle diameter, $\varepsilon$ is the porosity, and $\alpha$ is the permeability.

2.3. The Coupling of Discrete Phase and Porous Medium. The particles trajectory is tracked using the DPM model. The particle motion formula is on a Lagrange coordinate system. The particles motion formula of discrete phase is shown in (6) and the inertia force is considered.

$$
\frac{d u_{p}}{d t}=F_{D}\left(\mathrm{u}-u_{p}\right)+\frac{g_{x}\left(\rho_{p}-\rho\right)}{\rho_{p}}+F_{x}
$$

Here up is the velocity of particle. $\rho$ and $\rho_{p}$ are the densities of the continuous and discrete phase, respectively. $g_{x}$ is the acceleration of gravity in x direction; $g\left(\rho_{p}-\rho\right) / \rho_{p}$ represents the joint force of gravity and buoyancy when particle is moving; $F_{D}\left(u-u_{p}\right)$ is the air resistance acting on the particle in formula (6); and $F_{D}$ represents the resistance impacting on particles in unit-mass:

$$
\begin{aligned}
F_{D} & =\frac{18 \mu}{\rho_{p} d_{p}^{2}} \frac{C_{D} \operatorname{Re}}{24} \\
\operatorname{Re} & =\frac{\rho d_{p}\left|u_{p}-u\right|}{\mu} \\
C_{D} & =a_{1}+\frac{a_{2}}{\operatorname{Re}}+\frac{a_{3}}{\operatorname{Re}}
\end{aligned}
$$

$R_{e}$ is the relative Reynolds number and $C_{D}$ is the product ratio of fluid dynamic pressure. $F_{D}$ has impact 

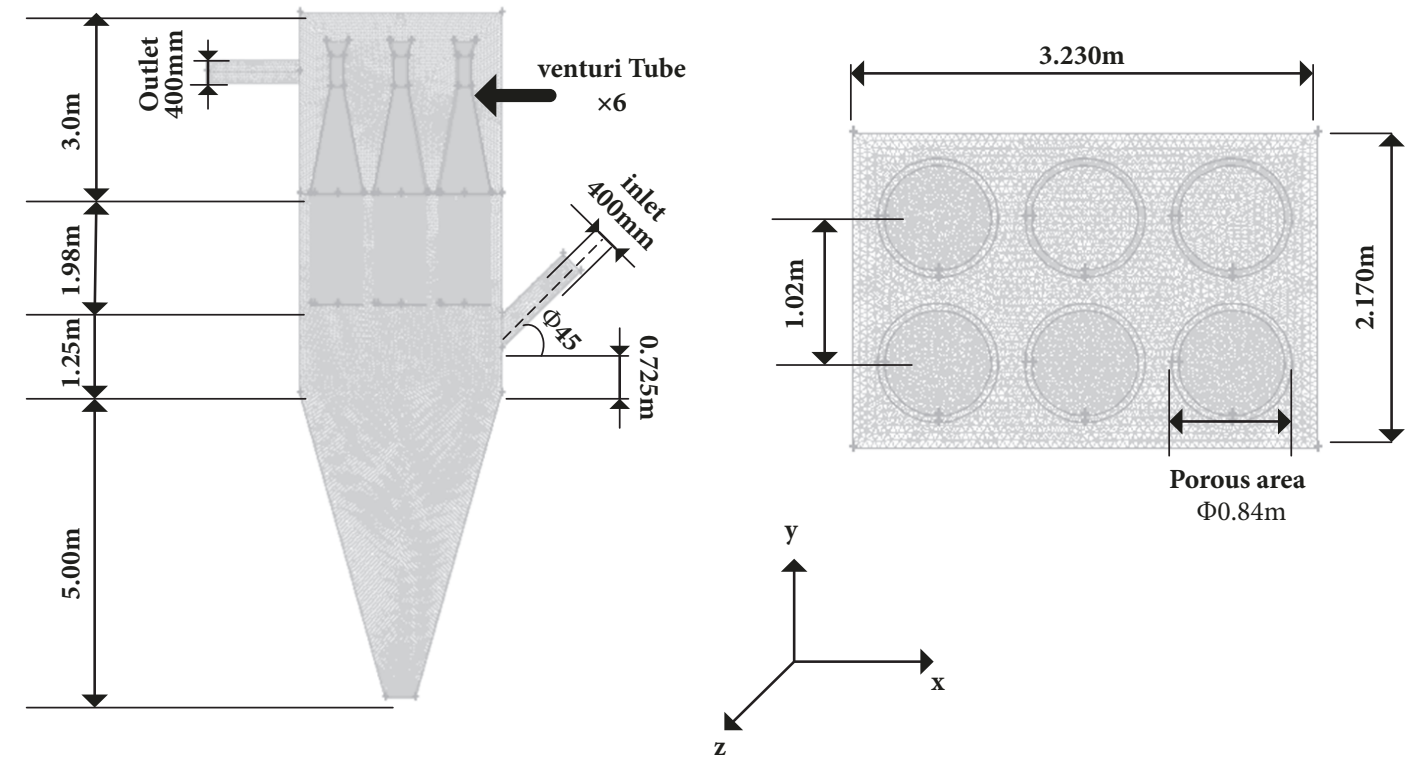

Figure 1: Calculation domain and grid arrangement.

on the projection which is the movement direction of the particles, namely, the drag force coefficient. $d_{p}$ is the particle diameter here. The control equations of coupling model in the continuous phase, discrete phase, and porous media $[17,18]$ are as follows:

$$
\begin{aligned}
& \varepsilon\left(\rho C_{p}\right)_{f} \frac{\partial T_{f}}{\partial t}+\left(\rho C_{p}\right) \varepsilon v \nabla T_{f} \\
& =\varepsilon \nabla\left(\mathrm{k}_{f} \nabla T_{f}\right)+(1-\varepsilon) \frac{6 h_{f}}{d}\left(\mathrm{~T}_{s}-\mathrm{T}_{f}\right) \\
& \left(\rho C_{s}\right) \frac{\partial T_{s}}{\partial t} \\
& =\nabla\left(\mathrm{k}_{s} \nabla T_{s}\right)+\frac{6 h_{f}}{d}\left(\mathrm{~T}_{s}-\mathrm{T}_{f}\right)+\rho_{s} \sum_{i}\left(-\nabla H_{i} \frac{\partial q_{i}}{\partial t}\right)
\end{aligned}
$$

$C_{p}$ and $C_{s}$ are the specific heat at constant pressure of the continuous and discrete phase, respectively; $h_{f}$ is the thermal coefficient of gas and solid phase; $k_{f}$ is axial heat transfer coefficient for the gas phase; ks is thermal conductivity coefficient for solid phase, unit for; $T_{f}$ and $\mathrm{Ts}$ are the temperature of gas and solid phase, respectively. Here transfer rate of mass equation is used:

$$
\frac{\partial \mathrm{q}_{i}}{\partial t}=k_{i}\left(\mathrm{q}_{i}^{*}-\mathrm{q}_{i}\right)
$$

Adsorption balance equations $[19,20]$ are as

$$
\begin{aligned}
\mathrm{q}_{i}^{*} & =\frac{K p_{i}}{1+\sum_{k=1}^{N} b_{k} P_{k}}, \\
K & =k_{i} \exp \frac{k_{2}}{T_{f}}, k_{3} \exp \frac{k_{4}}{T_{f}}
\end{aligned}
$$

$k_{i}$ is the mass transfer coefficient of the component $i$; qi is the adsorption quantity for solid phase of component $i$; $q *$ is the maximum adsorption amount for solid phase components.

\subsection{Principle and Simulation Conditions}

2.4.1. Separator Principle. The computational domain and grid arrangements of the separator are shown in Figure 1. The simulation domain consists of inlet, outlet, six Venturi tubes, and inferior vena including six filter parts. The diameters of inlet and outside are both $400 \mathrm{~mm}$. The angle between the centerlines of the inlet and the horizontal direction is $45^{\circ}$. There are six Venturi tubes in the separator. What is more, a back pulse made by vibration wave is used to reduce particles accumulated in filters elements. There are six filters in inferior vena which can be seen as porous mediums. Each filter has a diameter of $840 \mathrm{~mm}$ and a length of $1980 \mathrm{~mm}$.

The semi-implicit method for pressure-link equation (SIMPLE) algorithm is used as the numerical method. In computational fluid dynamics, the SIMPLE algorithm is widely used to solve the Navier-Stokes equations, and it has been extensively used to solve fluid flow and heat transfer problems $[21,22]$. A no-slip condition is set to the air phase on the walls. The simulation is started with the random generation of particles combined with gas phase in inlet. With the setting of gravitational force, lift force, and drag force settling, the particles enter into the inferior vena and pervade in the area of porous mediums. Finally, the part of the particles will sink into bottom; others will pass filter holes and reach the cavity Venturi tube and the export. In general we want to keep less particles away from passing through the porous area. Thus the filter will work for a longer time and not be broken easily. We hope more particles are blown down to the bottom of separator directly.

2.4.2. Simulation Conditions. Calculation domain is meshed by Gambit. The total number of meshes is 6,197,815; therefore, 
TABLE 1: The parameter settings of calculation.

\begin{tabular}{|c|c|c|}
\hline \multirow{6}{*}{ Gas phase } & Density(kg/m3) & 1.225 \\
\hline & $\operatorname{Viscosity}(\mathrm{kg} / \mathrm{m}-\mathrm{s})$ & $1.7894 \mathrm{e}-05$ \\
\hline & Reference Temperature(k) & 298.15 \\
\hline & Velocity Magnitude(m/s) & 20 \\
\hline & Turbulent Intensity(\%) & 10 \\
\hline & Hydraulic Diameter(m) & 0.4 \\
\hline \multirow{7}{*}{ Particles phase } & $\mathrm{Cp}($ Specific Heat $)(\mathrm{j} / \mathrm{kg}-\mathrm{k})$ & 1550 \\
\hline & Molecular Weight (kg/kg-mol) & 17.237 \\
\hline & Standard State Enthalpy(j/kg-mol) & $-5.601 e+07$ \\
\hline & Reference Temperature(k) & 298.15 \\
\hline & Velocity $(\mathrm{m} / \mathrm{s})$ & 20 \\
\hline & Turbulent Kinetic Energy & 0.8 \\
\hline & Momentum & 0.2 \\
\hline \multirow{5}{*}{ Porous area } & Porosity & 0.4 \\
\hline & face permeability(m2) & $1.19 \mathrm{e}-11$ \\
\hline & Porous permeability & 0.5 \\
\hline & Viscous Resistance & $8.4 \mathrm{e}+10$ \\
\hline & Inertial Resistance & $5.1 e+04$ \\
\hline
\end{tabular}

the time of calculation of the meshed model needs about 48 hours on a workstation (System is Windows 8 . The processor is Xeon 1231v3. The memory is $16 \mathrm{G}$ ). The calculation is done in Fluent 14.0 and the result is extracted by Tecplot 360 21013R1. The type of grid is chosen as tetrahedron structure. The grid density is increased appropriately in the six filter parts. The Jacobian value is positive.

In this study, we set medium volatile coal (Coal-Mv) as the particles of discrete phase in calculation. Compared with highly volatile coal (Coal-Hv) and low volatile coal (Coal$\mathrm{Lv})$, Coal-Mv is suitable for simulating most coal powders in working conditions [23]. The physical parameters are set according to the real property in Table 1.

The Rosin-Rammler model is used to describe the distribution of particle diameters in this work. The Rosin-Rammler model was first applied by Rosin-Rammler to describe a particle size distribution. The expression function of the Rosin-Rammler is described as

$$
F(d)=1-\exp \left[-\left(\frac{d}{c}\right)^{m}\right]
$$

where $\mathrm{F}$ is the distribution function of particles with a diameter of $\mathrm{d}$. $\mathrm{c}$ is the characteristic diameter which is always the average diameter of particles. The exponent $\mathrm{m}$ is uniformity constant $[24,25]$.

Inlet and outlet of the mode are mentioned in Figure 1. The area of filters is defined as the laminar zone porous. Other parameters in Ansys are chosen in Table 1. In Table 1, air is simulated as gas phase while particles phase is simulated as discrete phase. Filters are represented as porous area in separator. The physical parameters are set according to the real property. As to the velocity, diameter, energy, and momentum, average values are chosen to initialize and calculate. Momentum is the subrelaxation factor in Ansys 14.0 to reduce appropriately between the calculation results of the previous layer and the later one, which could avoid the nonlinear divergence induced by excessive difference during the iterative process.

In order to make result closer to reality, other options are set based on the real actual working conditions.

2.5. Simulation Result and Discussion. This section discusses the results of the simulation. The result positions of the three cross-sectional in porous region in Figure 2. Particle distribution in the three sections is shown in Table 2. We can find the following: (1) The particles enter into the inferior vena of separator, combined with continuous phase. Most particles cannot penetrate the porous media because there are viscous resistance and inertial resistance. (2) With the increase of time, particles slowly goes from import to inferior vena area. The number of particles gradually increases from bottom to top. Meanwhile, a large number of particles accumulated in the middle area. (3) Due to the fact that the particle phase is loaded continuously and the pressure is increased in the inferior vena, particles slowly enter into the filter which is close to inlet about the time of $23.4 \mathrm{~s}$. (4) The color of the particles indicates that the particles sink slowly to bottom of separator under the effect of gravity with time increases.

Table 3 shows the velocity distribution images in three cross-sectional. These sections illustrate the flow field of continuous phase under the influence of solid phase, and solid phase affects velocity distribution of the continuous phase. Take 60 speed values in each face to calculate the mean square error for each surface and it is shown in Table 4. Yi represents the mean square error of sample values in each cross section which is calculated as

$$
Y_{i}=\sqrt{\frac{1}{N} \sum_{i=1}^{N}\left(\mathrm{x}_{i}-\mu\right)^{2}}
$$




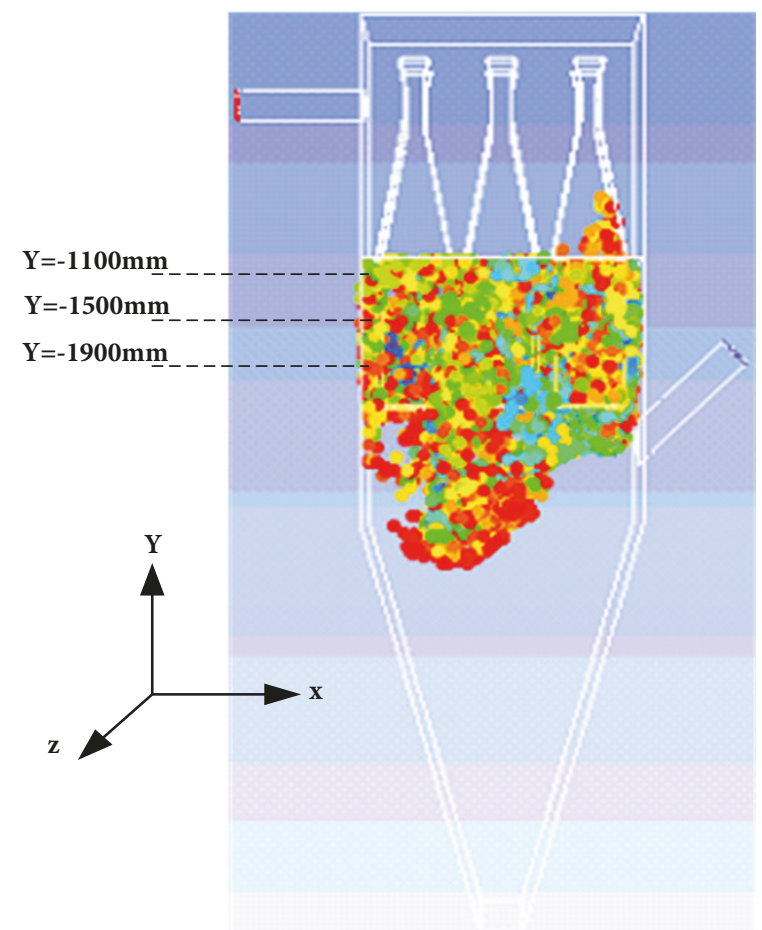

FiguRE 2: Three-dimensional particle distribution ( $\mathrm{t}=286 \mathrm{~s})$.

TABLe 2: Particle distribution at different times.

position Simulation result

$\mathrm{N}$ is the number of data point and $\mathrm{xi}$ is the value of each point. $\mu$ is the average value of all data points. The mean square error of each section is relatively large.

In engineering, the replacement of filter has a high cost and complicated operation. Therefore, we hope that most particles will not penetrate filter. A large number of particles accumulated in the middle area will cause filter to be partially stressed resulting in the aging rate of the filter, so particle should distribute uniformly in the filter to reduce the accumulation of particles partially in the filter. The particles are mainly affected by the continuous phase. Therefore, the goal of the separator structure optimization is to get a more homogeneous flow field in porous media zone. In our paper, the uniformity of flow field is evaluated by velocity and pressure. 
TABLE 3: The image of velocity distribution.

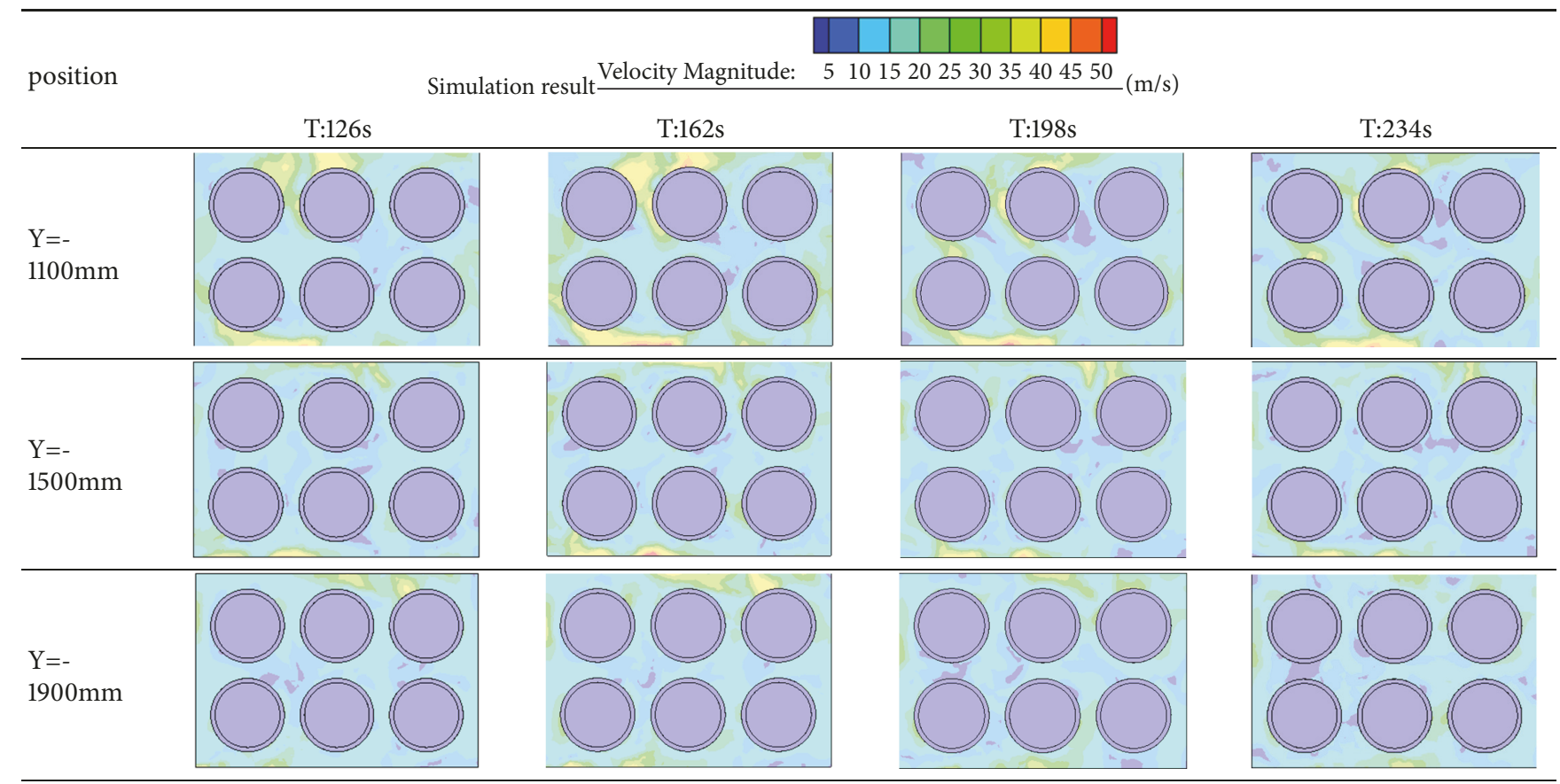

TABLE 4: The results of mean square error.

\begin{tabular}{lcccc}
\hline Position of section & $126 \mathrm{~s}$ & $162 \mathrm{~s}$ & $198 \mathrm{~s}$ & $234 \mathrm{~s}$ \\
$-1100 \mathrm{~mm}$ & 10.877 & 9.957 & 9.073 & 8.838 \\
$-1500 \mathrm{~mm}$ & 7.886 & 7.852 & 8.083 & 5.441 \\
$-1900 \mathrm{~mm}$ & 6.777 & 6.777 & 5.685 & 6.230 \\
\hline
\end{tabular}

\section{The Optimizations of Structure for Separator}

A separator model is given by an enterprise in China. Our purpose is to improve parameters about inlet and filters based on CFD. As to a separator, performance is always decided by the distribution of velocity and pressure. In ideal status, a uniform distribution of flow field means that the particle will contact the filters equivalently; therefore, all the filters paly the same role. Otherwise, particles will focus on some parts of few filters which will be damaged easily. These filters may be blocked by too much concentrated particles, while an unfair distribution also means that some area of filters does not work at all. For these reasons, mean square errors of velocity and pressure are used to evaluate the result of optimization, and a smaller value means a better performance.

As to a given separator in specific working conditions, the mainframe cannot be changed, and the filter is already made according to standard; therefore, their sizes always cannot be changed. Inlet is a key part to decide the distribution of fluid filed; thus both the position and inclination angle of inlet are chosen to optimize. In the inner of separator, the utilization rate of filters is decided by the installation site directly, since it is so, it cannot be neglected that the distance between two filters in the direction- $\mathrm{Z}$ in Figure 1. The distance- $\mathrm{X}$ is neglected here to simplify the process since its result is similar to distance in direction- $Z$.

Based on the reasons above, three parameters are selected to optimize the model according to the mean square error from distribution of both velocity and pressure. Taguchi method is used to realize the target.

3.1. The Theory of Taguchi Method. The basic principle of the Taguchi method, avoiding the blindness of traverse type analysis, is the inspection of the effectiveness from the experimental variables which is the synthesis procedure conducted by orthogonal array with minimum number of experiments $[15,26]$. This approach can obtain effectively the contribution rate and weight relations from various elements of target. The method can be used to realize the optimization and reduce test times compared to exhaustion method. The main steps of structure optimization for separator are as follows: (1) Determining the key elements of the research object, select all elements of the plan. (2) Design the orthogonal table for all combination schemes according to the relationship between elements. (3) Select the orthogonal schemes and calculate each of them. (4) According to the results, get the order of weight for all selected elements based on the contribution rate.

3.2. The Design of Orthogonal Table. In the study based on Taguchi method, to represent three parameters, we take the angle between the inlet axis and the horizontal direction as factor $\mathrm{A}$, the distance between the inlet and bottom of filter as factor $\mathrm{B}$, and the distance of two filters in direction- $\mathrm{Z}$ as factor C. Control factors and its levels are designed and shown in Table 5.

Factor $\mathrm{A}$ is often between $0^{\circ}$ to $45^{\circ}$ in actual equipment, thus $0^{\circ}, 45^{\circ}$, and $22.5^{\circ}$ are chosen. As to factor B and factor 
TABLE 5: The statistics of element.

\begin{tabular}{lccc}
\hline Factor & Level 1 & Level 2 & Level 3 \\
\hline A (degree) & 45 & 22.5 & 0 \\
B (m) & 1.267 & 0.725 & 0.200 \\
C (m) & 1.220 & 1.020 & 0.820 \\
\hline
\end{tabular}

TABLE 6: The proof of the orthogonal.

\begin{tabular}{llllllc}
\hline case & $\mathrm{A}$ & $\mathrm{B}$ & $\mathrm{C}$ & $\mathrm{AB}$ & $\mathrm{BC}$ & $\mathrm{CA}$ \\
\hline 1 & + & + & + & + & + & + \\
2 & + & - & - & - & + & - \\
3 & + & 0 & 0 & 0 & 0 & 0 \\
4 & - & + & - & - & - & + \\
5 & - & - & 0 & + & 0 & 0 \\
6 & - & 0 & + & 0 & 0 & - \\
7 & 0 & + & 0 & 0 & 0 & 0 \\
8 & 0 & - & + & 0 & - & 0 \\
9 & 0 & 0 & - & 0 & 0 & 0 \\
Total & 0 & 0 & 0 & 0 & 0 & 0 \\
\hline
\end{tabular}

$\mathrm{C}$, the extreme values and their medium value are taken into consideration, ranging from the highest and lowest position. Different values are noted as different levels in Taguchi method as shown in Table 5.

Table 6 is the orthogonal table for Taguchi analysis with factors A, B, and C. A correct orthogonal table needs to fulfill with following two conditions: (1) In a column, the numbers of different values should be the same. (2) The same levels have the same frequency in every two columns.

$\mathrm{L}_{9}$ orthogonal array is used here; it contains nine tests, three levels, and three variables ( $\mathrm{L}$ means Latin square and 9 means the number of cases). The orthogonal table is recorded in Table 7 and it can be used as experiment scheme.

3.3. The Analysis of the SNR. In Taguchi method, the ratio of signal to noise (SNR) is always used as the key value to evaluate the performance of each experiment in Taguchi analysis. In this study, the target is to find smaller mean square errors; thus SNR can be expressed as (16). A bigger value of SNR is needed since it is negatively correlated with Y [27].

$$
\eta=-10 \lg \frac{1}{n}\left(\sum_{i=1}^{n} Y_{i}^{2}\right)
$$

$\eta$ represents the SNR. $n$ is the number of replications. Since it is expected to be smaller that both the mean square errors of velocity and pressure, a smaller value is what we needed. In Taguchi method, the ratio of signal to noise (SNR) is used instead of mean square errors as SNR can expand the range of smaller values. In (16), $Y_{i}^{2}$ is used to eliminate the negative values and an average is calculated. A logarithmic relation can amplify the average of $Y_{i}^{2}$ when it is under 1 ; this means the contrast ratio for smaller values is increased and it is benefit to our optimization based on mean square errors. Then the result is multiplied by 10 to observe easily. Finally, an opposite number is chosen and that means that a smaller mean square
TABLE 7: The orthogonal table of separator.

\begin{tabular}{llll}
\hline case & A & B & C \\
\hline 1 & A1 & B1 & C1 \\
2 & A1 & B2 & C2 \\
3 & A1 & B3 & C3 \\
4 & A2 & B1 & C2 \\
5 & A2 & B2 & C3 \\
6 & A2 & B3 & C1 \\
7 & A3 & B1 & C3 \\
8 & A3 & B2 & C2 \\
9 & A3 & B3 & C1 \\
\hline For example, A1 stands for factor A with Level 1. &
\end{tabular}

error has a bigger SNR as (16). We can find that SNR can expand the range of smaller values with logarithmic relation and describe the result more clearly. In another way, SNR is more sensitive to a small value and that is what we care; thus we take SNR instead of Y. From the explanation above, a better result has a bigger SNR as to (16).

The SNR, a function of noise, is a good performance measure for robustness, is a good performance measure for robustness. Thus, it is expected that the results of statistically significant should represent the optimal combination of kinematic parameters [28]. The $\eta$ is used to evaluate the fluctuation of mean square error about combination parameters in the article.

Values of $Y$ and $\eta$ from 180 data points on three sections of each model are calculated. 9 cases in Table 7 are calculated and the results are recorded in Tables 8 and 9. Table 8 shows the result with velocity while Table 9 with pressure.

Then the contribution rate is expressed as (17). It reflects the importance of different factors to $\eta . \mathrm{z}$ is the $z$ th control factors, namely, the factors in Table $4 . \eta_{\max } z^{-} \eta_{\min } z$ is the range of different factors. $m$ is the number of levels of a factor. Contribution rates are recorded in Tables 10 and 11.

$$
C R_{z}=\frac{\eta_{\max z}-\eta_{\min z}}{\sum_{z=1}^{m}\left(\eta_{\max z}-\eta_{\min z}\right)} \times 100 \%
$$

From Table 10, A and B play important roles in determine the uniform of velocity, because they both have a contribution over 39\%. The influence of $\mathrm{C}$ is much smaller. From Table 11, it can be found that $\mathrm{C}$, over $56 \%$, is considered to be the most important factor to distribution of pressure and $\mathrm{A}$ is less important with $41 \%$, while factor B has almost no impact on pressure with $2.184 \%$. In the work, we must select the factors with contribution rate.

In order to obtain better parameters of the separator, both distributions are considered. The values of $\eta$ according to factors are shown in Figures 3 and 4 . Target values of $\eta$ are bigger ones, with contribution rate; the best scheme is $\mathrm{A} 2 \mathrm{~B} 2 \mathrm{C} 2$ according to Figure 3. As to the effective of pressure, $\mathrm{A} 2 \mathrm{~B} 2 \mathrm{C} 2$ is still the best scheme. Although it comes from different physical quantities, the result is the same.

It can be seen that there is a positive correlation between the distribution of velocity and pressure. A better scheme 
TABLE 8: The results about velocity.

\begin{tabular}{|c|c|c|c|c|c|}
\hline case & $\mathrm{A}$ (degree) & $\mathrm{B}(\mathrm{m})$ & $\mathrm{C}(\mathrm{m})$ & $\mathrm{Y}$ & $\eta$ \\
\hline 1 & 45 & 1.267 & 1.220 & 3.000 & -9.542 \\
\hline 2 & 45 & 0.725 & 1.020 & 1.052 & -0.440 \\
\hline 3 & 45 & 0.200 & 0.820 & 1.895 & -5.552 \\
\hline 4 & 22.5 & 1.267 & 1.020 & 1.338 & -2.529 \\
\hline 5 & 22.5 & 0.725 & 0.820 & 0.677 & 3.388 \\
\hline 6 & 22.5 & 0.200 & 1.220 & 0.121 & 18.344 \\
\hline 7 & 0 & 1.267 & 0.820 & 1.687 & -4.542 \\
\hline 8 & 0 & 0.725 & 1.020 & 0.181 & 14.846 \\
\hline 9 & 0 & 0.200 & 1.220 & 0.790 & 2.047 \\
\hline
\end{tabular}

TABLE 9: The results about pressure.

\begin{tabular}{lccccc}
\hline case & $\mathrm{A}($ degree $)$ & $\mathrm{B}(\mathrm{m})$ & $\mathrm{C}(\mathrm{m})$ & $\mathrm{Y}$ & \multicolumn{1}{c}{$\eta$} \\
\hline 1 & 45 & 1.267 & 1.220 & 38.132 & -31.626 \\
2 & 45 & 0.725 & 1.020 & -24.113 \\
3 & 45 & 0.200 & 0.820 & 43.186 & -32.707 \\
4 & 22.5 & 1.267 & 1.020 & 3.895 & -11.810 \\
5 & 22.5 & 0.725 & 0.820 & 40.566 & -32.163 \\
6 & 22.5 & 0.200 & 1.220 & -6.042 & -35.801 \\
7 & 0 & 1.267 & 0.820 & 3.405 & -10.642 \\
8 & 0 & 0.725 & 1.020 & 31.813 \\
9 & 0 & 0.200 & 1.220 & -30.052 \\
\hline
\end{tabular}

TABLE 10: The contribution of factors based on velocity.

\begin{tabular}{ccccc}
\hline & & $\mathrm{A}$ & $\mathrm{B}$ & $\mathrm{C}$ \\
\hline \multirow{3}{*}{$\mathrm{SNR}$} & 1 & -5.178 & -5.528 & 3.616 \\
& 2 & 6.401 & 5.931 & 3.959 \\
& 3 & 4.117 & 4.947 & -2.235 \\
\hline \multicolumn{2}{c}{ Range } & 11.579 & 11.459 & 6.194 \\
\hline \multicolumn{2}{c}{ Contribution Rate } & $39.611 \%$ & $39.200 \%$ & $21.189 \%$ \\
\hline
\end{tabular}

TABLE 11: The contribution of factors based on pressure.

\begin{tabular}{ccccc}
\hline & & $\mathrm{A}$ & $\mathrm{B}$ & $\mathrm{C}$ \\
\hline \multirow{3}{*}{ SNR } & 1 & -29.482 & -26.123 & -22.626 \\
& 2 & -16.725 & -22.306 & -15.522 \\
& 3 & -25.209 & -22.987 & -33.267 \\
\hline \multicolumn{2}{c}{ Range } & -12.757 & -0.681 & -17.745 \\
\hline \multicolumn{2}{c}{ Contribution Rate } & $40.910 \%$ & $2.184 \%$ & $56.906 \%$ \\
\hline
\end{tabular}

can be obtained with Taguchi method to get a uniform distribution of fluid filed in inner part.

3.4. Result. From the result above, A2B2C2 is considered as the best scheme. The mean square errors of velocity and pressure are shown in Figures 5 and 6, respectively.

The mean square error of the 10th scheme is close to the optimal, as well as the case of 6 th scheme and the case of 8 th scheme. The most obvious characteristic of the three cases is the smaller angle between import and horizontal. Therefore, control factor A has great influence for the flow field uniformity in the filter sections. We can draw the conclusion that the Taguchi method can also evaluate the importance of a single factor.

The Taguchi methods can not only evaluate the importance of elements and also evaluate the rate of contribution of the single factor. This method applied in structure design can be refined and extended in a follow-up study such as increasing the number of parameters and the degree of level factors. A better scheme can be got to reduce the cost of design and manufacture through the analysis and calculation.

\section{Conclusion}

In this paper, a separator is simulated with CFD, and key parameters of a separator are optimized with Taguchi method. The results in this paper are as follows:

(1) The process of movement and diffusion of particles is obtained. The accumulation areas of particle are found, and the causes of accumulation are analyzed through the distribution figures. An evaluation for the structure of the original separator is taken.

(2) Taguchi method is introduced to take orthogonal experiment. It can reduce 27 schemes to 9 . Then the weights of key parameters are determined through the contribution rate and it can be known which parameter has a bigger influence on separator

(3) The optimal scheme, namely, A2B2C2, is determined according to the results of a bigger SNR value. It is verified by numerical simulation and found that mean square error of distributions from both velocity and pressure is close to the minimum. The optimization of the structure is realized. 


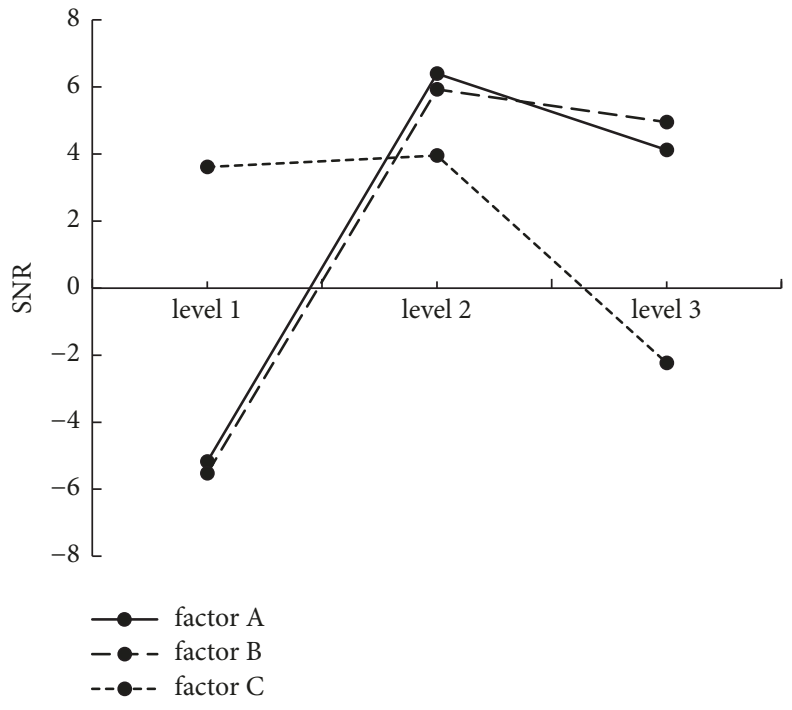

FIGURE 3: SNR according to velocity.

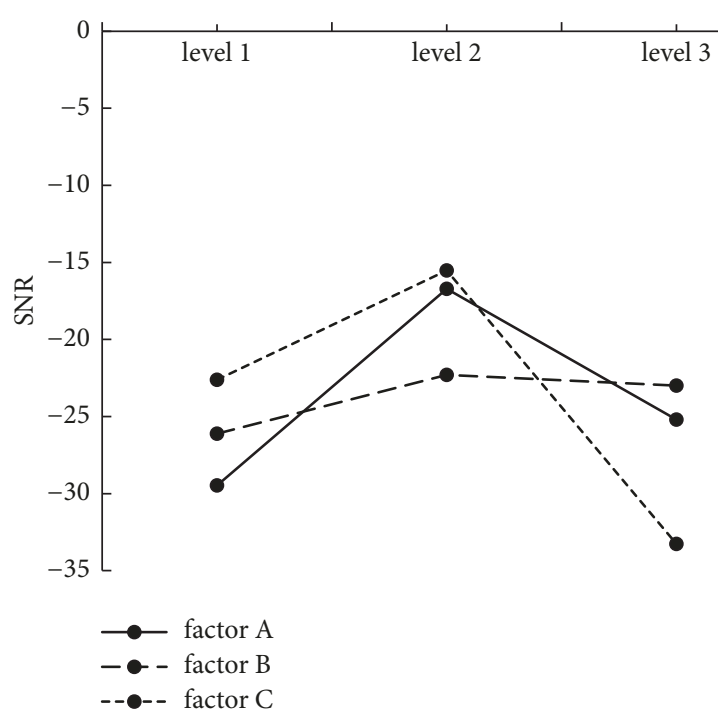

FIGURE 4: SNR according to pressure.

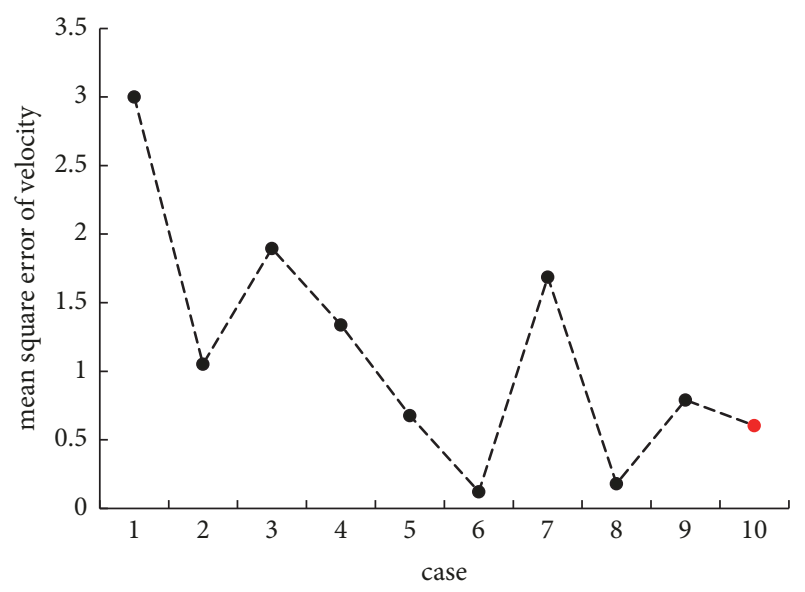

Figure 5: The mean square error of velocity in all cases.

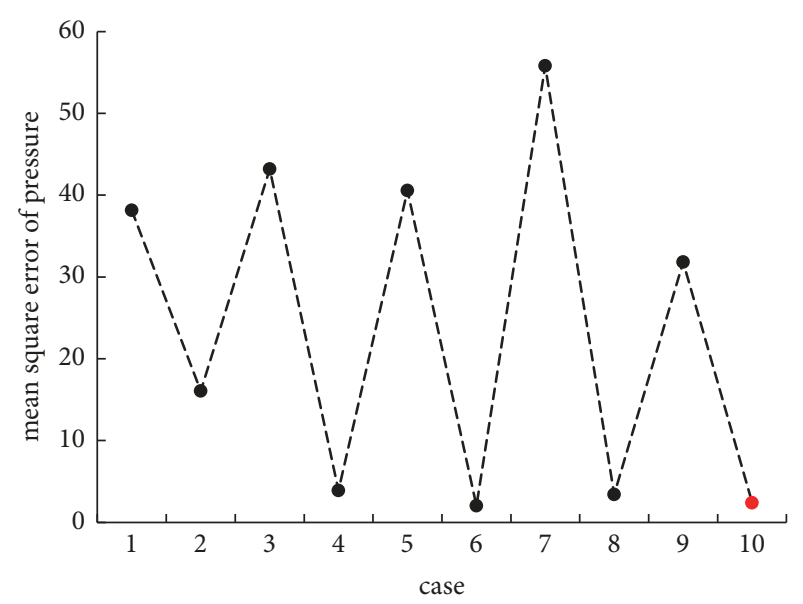

FIGURE 6: The mean square error of pressure in all cases.

In this paper a theoretical foundation and effective means has been provided and help in extending the knowledge on the research of optimization design in separator.

\section{Data Availability}

The data used to support the findings of this study are included in the supplementary material file.

\section{Conflicts of Interest}

The authors declare that they have no conflicts of interest.

\section{Acknowledgments}

This work was supported by the National Natural Science Foundation of China (Grant 51876175), the Innovative Talents 
Promotion Project of Shaanxi, China (Grant 2018KJXX067), and the Tianjin Municipal Science and Technology Foundation (no. 14JCYBJC43200).

\section{Supplementary Materials}

The data is extracted from the postprocessing software Tecplot. There are a total of 9 cases with 120 speed values for each case. The mean square error is obtained by the 120 data of each case. SNR is obtained from the formula in Table 5 of the article. The o data processing in other forms have been clearly stated in paper. The file data are used to support the findings of this study. (Supplementary Materials)

\section{References}

[1] Y. Xiong, "High moisture lignite coal burning power generation integrated drying technology," Boiler Technology, pp. 46-49, 2006.

[2] J. A. M. Kuipers, K. J. Van Duin, F. P. H. Van Beckum, and W. P. M. Van Swaaij, "A numerical model of gas-fluidized beds," Chemical Engineering Science, vol. 47, no. 8, pp. 1913-1924, 1992.

[3] H. T. Bi and J. Li, "Multiscale analysis and modeling of multiphase chemical reactors," Advanced Powder Technology, vol. 15, no. 6, pp. 607-627, 2004.

[4] Y. Tsuji, T. Tanaka, and T. Ishida, "Lagrangian numerical simulation of plug flow of cohesionless particles in a horizontal pipe," Powder Technology, vol. 71, no. 3, pp. 239-250, 1992.

[5] Y. X. Wu, X. Wang, and C. B. Ching, "Computational fluid dynamics simulation of the adsorption separation of three components in high performance liquid chromatography," Chromatographia, vol. 55, no. 7-8, pp. 439-445, 2002.

[6] H. Boysen, G. Wozny, and T. Laiblin, "CFD simulation of preparative HPLC columns with consideration of nonlinear isotherms," Chemical Engineering \& Technology, vol. 26, no. 6, pp. 651-655, 2003.

[7] Y. Liming, X. Zhou, J. Yang et al., "Numerical Simulation of Water and Sediment Movement in Screen Filter Based on Coupled CFD-DEM," Transactions of the Chinese Society for Agricultural Machinery, vol. 3, pp. 303-308, 2018.

[8] B. Wang, D. L. Xu, K. W. Chu, and A. B. Yu, "Numerical study of gas-solid flow in a cyclone separator," Applied Mathematical Modelling, vol. 30, no. 11, pp. 1326-1342, 2006.

[9] T. Zhao, K. Liu, Y. Cui, and M. Takei, "Three-dimensional simulation of the particle distribution in a downer using CFDDEM and comparison with the results of ECT experiments," Advanced Powder Technology, vol. 21, no. 6, pp. 630-640, 2010.

[10] M. Haim, Y. Weiss, H. Kalman, and A. Ullmann, "The effect of the inlet conditions on the numerical solutions of particle-gas flows," Advanced Powder Technology, vol. 14, no. 1, pp. 87-110, 2003.

[11] X. Gao, T. Li, A. Sarkar, L. Lu, and W. A. Rogers, "Development and validation of an enhanced filtered drag model for simulating gas-solid fluidization of Geldart A particles in all flow regimes," Chemical Engineering Science, vol. 184, pp. 33-51, 2018.

[12] J. A. Pamanes, J. P. Montes, E. Cuan, and G. F. Rodriguez, "Optimal placement and synthesis of a 3R manipulator," in Proceedings of the International Symposium on Robotics and Automation, Monterrey, Mexico, 2000.
[13] G. Taguchi, Introduction to Quality Engineering Designing: Quality into Products and Processes, Asian Productivity Organization, Tokyo, Japan, 1986.

[14] G. Taguchi, Studies on Mathematical Statistics for Quality Control [Doctoral thesis], Kushu University, Japan, 1962.

[15] B. K. Rout and R. K. Mittal, "Tolerance design of robot parameters using Taguchi method," Mechanical Systems and Signal Processing, vol. 20, no. 8, pp. 1832-1852, 2006.

[16] T. Mothilal, K. Pitchandi, V. Velukumar, and K. Parthiban, "CFD and statistical approach for optimization of operating parameters in a tangential cyclone heat exchanger," Journal of Applied Fluid Mechanics, vol. 11, no. 2, pp. 459-466, 2018.

[17] A. Bejan and D. A. Nield, Convection in Porous Media, Springer, New York, NY, USA, 3rd edition, 2006.

[18] T. L. Dantas, F. M. Luna, I. J. Silva et al., "Carbon dioxide-nitrogen separation through pressure swing adsorption," Chemical Engineering Journal, vol. 172, no. 2-3, pp. 698-704, 2011.

[19] E. Glueckauf, “Theory of chromatography. Part 10. Formula for diffusion into spheres and their application to chromatography," Transactions of the Faraday Society, vol. 51, pp. 1540-1551, 1955.

[20] S. U. Rege and R. T. Yang, "Limits for Air Separation by Adsorption with LiX Zeolite," Industrial \& Engineering Chemistry Research, vol. 36, no. 12, pp. 5358-5365, 1997.

[21] J. C. Tannehill, R. H. Pletcher, and D. A. Anderson, Computational Fluid Mechanics Foundation and Its Applications, Taylor \& Francis Inc, UK, 2nd edition, 1997.

[22] L. Mangani and C. Bianchini, "Heat transfer applications in turbomachinery," in Proceedings of the Open FOAM International Conference, Florence, Italy, 2007.

[23] T. Wigmans, H. Haringa, and J. A. Moulijn, "Role of the influence of potassium during pyrolysis of medium volatile coal," Fuel, vol. 63, no. 6, pp. 870-872, 1984.

[24] P. Rosin and E. Rammler, "The Laws Governing the Fineness of Powdered Coal," Journal of the Institute of Fuel, vol. 7, pp. 29-36, 1933.

[25] N. C. Sagias and G. K. Karagiannidis, "Gaussian class multivariate Weibull distributions: theory and applications in fading channels," Institute of Electrical and Electronics Engineers Transactions on Information Theory, vol. 51, no. 10, pp. 3608-3619, 2005.

[26] Q. Zou, Y. Xu, and C. Zheng, "Optimization of Structural Parameters of Drilling slotting Integration Nozzle Based on Taguchi Method," Coal Mine Machinery, vol. 33, no. 10, pp. 1719, 2012.

[27] Z.-B. Yang, M. Radzienski, P. Kudela, and W. Ostachowicz, "Two-dimensional Chebyshev pseudo spectral modal curvature and its application in damage detection for composite plates," Composite Structures, vol. 168, pp. 372-383, 2017.

[28] L. Wang, C. Wang, W. Du et al., "Parameter optimization of a four-legged robot to improve motion trajectory accuracy using signal-to-noise ratio theory," Robotics and Computer-Integrated Manufacturing, vol. 51, pp. 85-96, 2018. 


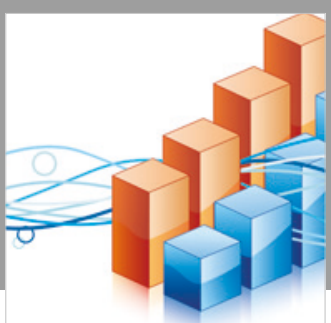

Advances in

Operations Research

\section{-n-m}
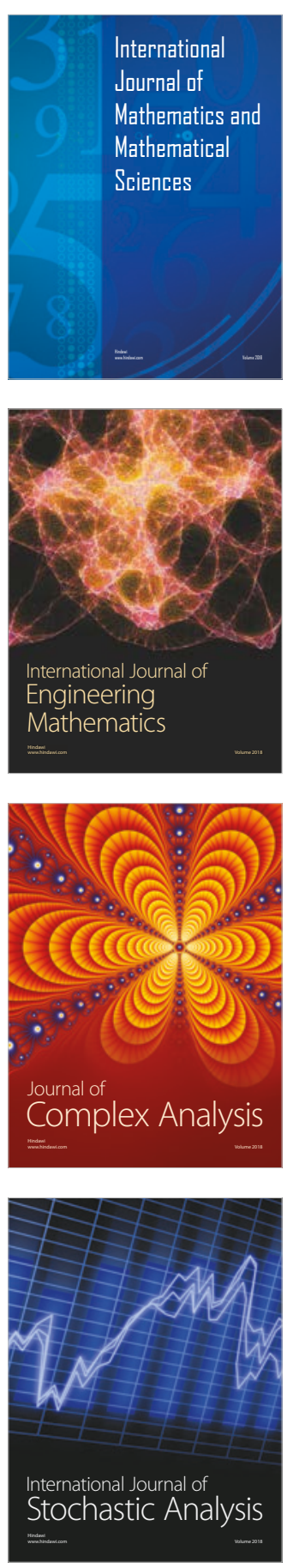
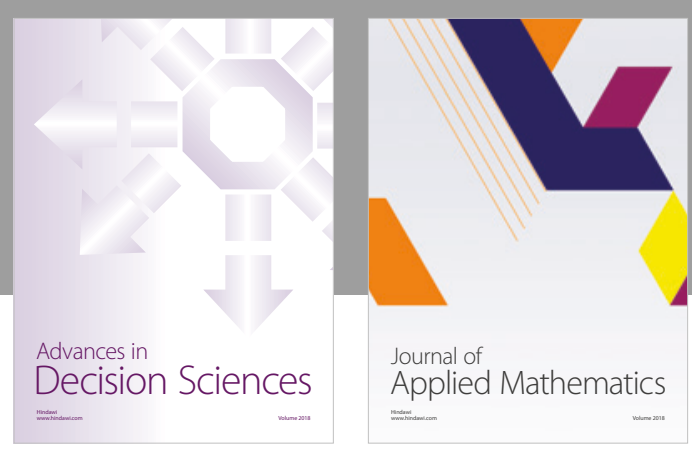

Journal of

Applied Mathematics
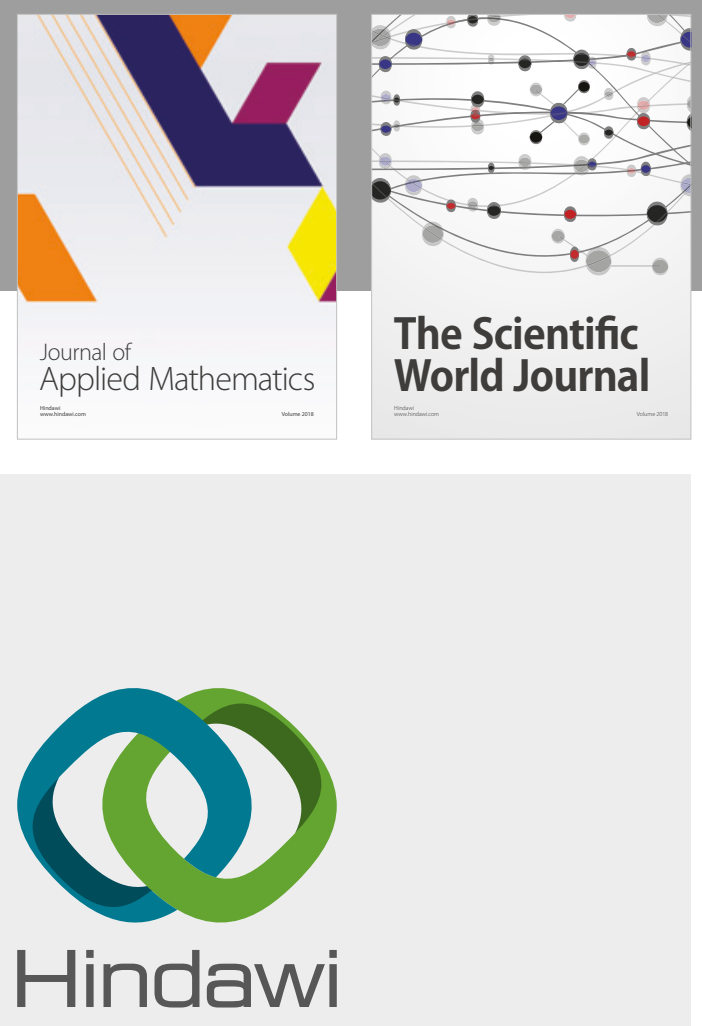

Submit your manuscripts at

www.hindawi.com

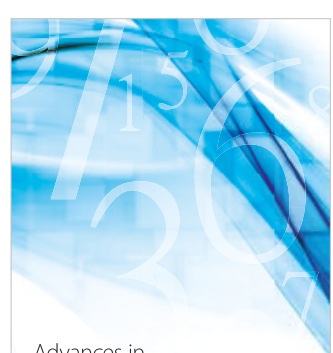

Advances in
Numerical Analysis
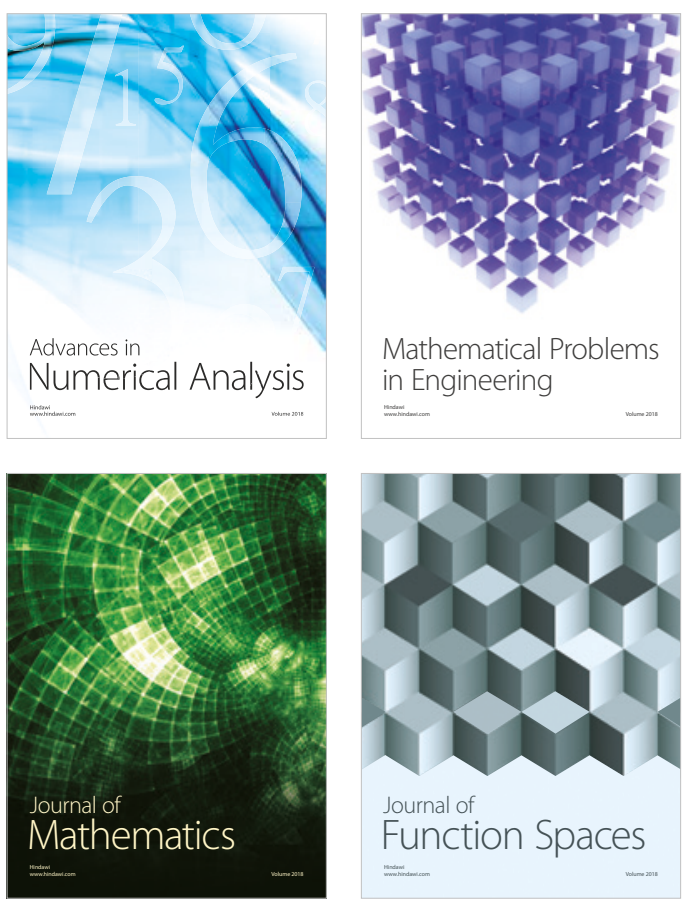

Mathematical Problems in Engineering

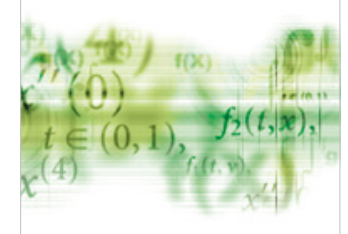

International Journal of

Differential Equations

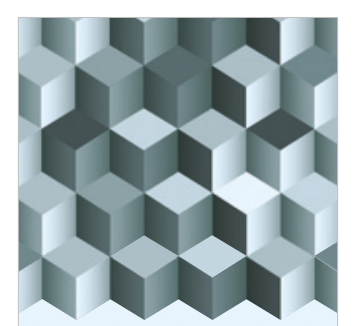

Journal of

Function Spaces

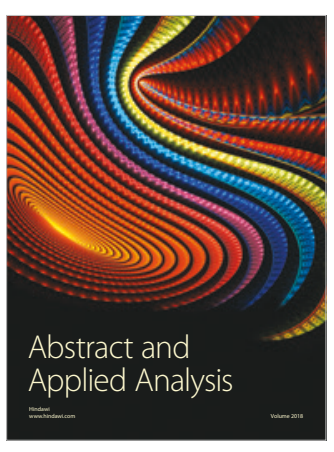

The Scientific

World Journal

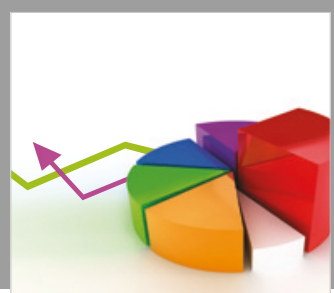

Journal of

Probability and Statistics
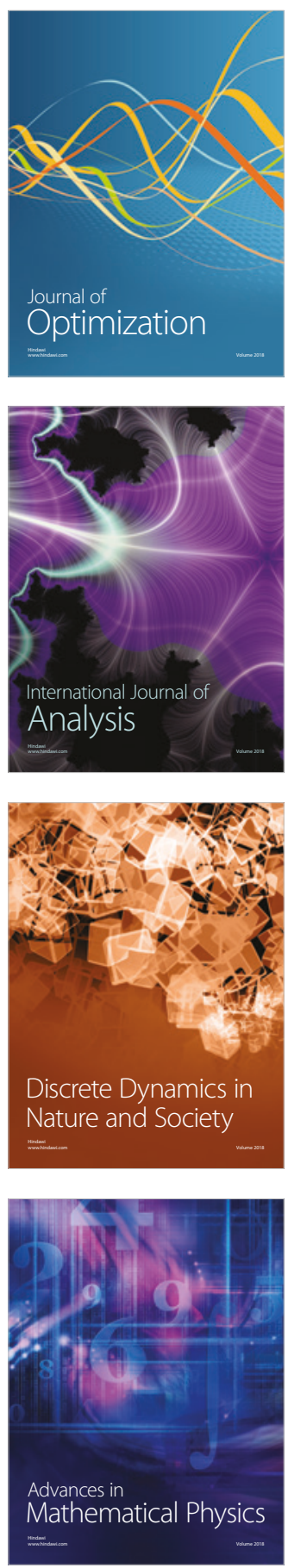